\title{
Spatial distance of target locations affects the time course of both endogenous and exogenous attentional deployment
}

\author{
Frederik Geweke ${ }^{1}$, Emilia Pokta ${ }^{1}$, Viola Störmer ${ }^{2}$ \\ ${ }^{1}$ Department of Psychology, University of California San Diego \\ ${ }^{2}$ Psychological and Brain Science Department, Dartmouth College
}

Please address correspondence to:

Viola Störmer

Department of Psychological and Brain Sciences

Dartmouth College

Email: viola.s.stoermer@dartmouth.edu

RUNNING HEAD: temporal limits of exogenous and endogenous attentional deployment 


\begin{abstract}
Spatial attention can be deployed exogenously, based on salient events in the environment, or endogenously, based on current task goals. Numerous studies have compared the time courses of these two types of attention, and have demonstrated that exogenous attention is fast and transient and endogenous attention is relatively slow but sustained. In the present study we investigated whether and how the temporal dynamics of exogenous and endogenous attention differ in terms of where attention is deployed in the visual field, in particular at locations nearby or far from fixation. Across a series experiments, we measured attentional shift times for each type of attention, and found overall slower deployment of endogenous relative to exogenous attention, in line with previous research. Importantly, we also consistently found that it takes longer to deploy attention at more distant locations relative to nearby locations, regardless of how attention was instigated. Overall, our results suggest that the temporal limits of attentional deployment across different spatial distances are similar for exogenous and endogenous attention, pointing to shared constraints underlying both attentional modes.
\end{abstract}

KEYWORDS: visual attention, exogenous attention, endogenous attention, attentional shift times 


\section{Significance statement}

This study addresses a seminal question in attention research, namely how long it takes for spatial attention to shift from one location to another, and whether it matters how close or far these locations are to each other. The results show that it takes longer for attention to be deployed to locations at further distances in the visual field, regardless of whether attention is triggered by salient events in the environment, or is oriented based on top-down goals. This demonstrates that different modes of attention share similar temporal constraints when deploying attention to new locations. 


\section{Introduction}

Attention can be deployed based on current goals, for example when focusing on the side of the road to look for a street sign, and attention can also be grabbed reflexively by a sudden event in the environment, such as the flash of a traffic light (LaBerge, 1995; Wright \& Ward, 2008). These modes of spatial attention, commonly referred to as endogenous and exogenous attention, respectively, both result in behavioral benefits at the attended location, reflected in faster response times and/or higher accuracy for attended relative to unattended information (Posner, 1980; for a review, see Carrasco, 2011). Neural data has shown that the large-scale brain networks involved in each type of attention are in part overlapping (Peelen, et al., 2004; Serences \& Yantis, 2007), and recent results indicate that endogenous and exogenous attention cues can produce similar effects in early visual cortex prior to the onset of a target (Keefe \& Störmer, 2020). Thus, there are many similarities in how exogenous and endogenous attention affect behavioral and neural processing. However, there are also clear differences and much research has argued for two separate attention systems (e.g., Hahn et al., 2006; Chica, et al., 2013). Perhaps most striking are the differences in their temporal dynamics: exogenous attention is deployed quickly and transiently, while endogenous attention is deployed on a relatively slower time scale (Müller \& Rabbit, 1989; Nakayama \& Mackeben, 1989; Keefe \& Störmer, 2020). These differences in time course have been interpreted as a hallmark distinction between exogenous and endogenous attention (Egeth \& Yantis, 1997; Eriksen \& Hoffman, 1972; Jonides, 1981; Nakayama \& Mackeben, 1989; Posner, 1980).

What can these general differences in timing tell us about the processes underlying the deployment of exogenous and endogenous spatial attention? One possibility is that the 
allocation of spatial attention - i.e., shifting attention from one location to the other - is implemented by different mechanisms during exogenous and endogenous orienting (Corbetta \& Shulman, 2002; Hopfinger \& West, 2006; Hahn et al., 2006; Ling \& Carrasco, 2006; Barbot, et al., 2012), and that these different mechanisms require different amounts of time to be executed. Another possibility, however, is that the temporal differences are the result of processes not related to the orienting of attention itself, but rather to differences in cue or target processing. In the laboratory, exogenous attention is usually modeled by presenting a salient visual stimulus briefly at a target location shortly before the onset of a target (the attention cue), and by measuring responses to the target separately for when the target is presented at the same location as the cue (valid trials) relative to when the target is presented at another location (invalid trials; e.g., Posner, 1980; Fuller, et al.,, 2009; Störmer \& Alvarez, 2016). Endogenous attention, on the other hand, is usually tested in an experimental setting by presenting symbolic cues at the center of the screen instructing participants to attend to a particular location. For example, a central arrow or a line pointing leftwards indicate to a participant to direct attention to the left side of the screen in preparation of an impending target stimulus. To ensure participants are following these instructions, the cues often predict the target location at a certain probability (e.g., with $80 \%$ validity; Posner, 1980). Using these types of tasks, cue benefits for target processing are often found $~ 100 \mathrm{~ms}$ after cue presentation in the exogenous case, but only 300-500 ms after endogenous cues (e.g., Nakayama \& Mackeben, 1989; Müller\& Rabbit, 1989). These time differences, however, are not necessarily driven by differences in the time course of shifting attention to the target location, but can also be explained by differences in processing the spatial cues. Specifically, a symbolic 
cue needs to be interpreted and mapped to the physical target location, but these types of planning and mapping processes are not required for typical exogenous cues that usually appear at the target location itself (Hazlett \& Woldorff, 2004). Thus, comparing the absolute time differences between exogenous and endogenous spatial attention may tell us little about the underlying processes related to the of shifting attention itself - in particular whether they operate on different time scales. A much more informative approach may be to examine how attentional shift times vary depending on where in the visual field attention is deployed - in particular, whether the time course of exogenous and endogenous attention differs in terms of the spatial distance attention travels.

There is ample research examining how long it takes for spatial attention to shift to different locations in the visual field. While some studies have found that the spatial distance affects the time course of attention, showing that it takes longer to move attention to more distant locations using either central symbolic (e.g., Rizzolatti, et al., 1987; Shulman, et al.,; 1979) or peripheral cues (Egly \& Homa, 1991; Tsal \& Mevorach, 1991), other studies found no effect of spatial distance on timing for either cue (symbolic cues: Sperling \& Weichselgartner, 1995; Remington \& Pierce, 1984; both symbolic or peripheral cues: Cheal \& Lyon, 1989). These mixed results may in part be due to different stimuli being used, thus making it difficult to disentangle processes related to cue interpretation, shifting attention, or target processing. A more recent study used an elegant subtraction method to control for differences in cue processing time between endogenous and exogenous attention and found that spatial distance affected endogenous shifts of attention but much less so exogenous shifts of attention (Chakravarthi \& van Rullen, 2011, see also Carlson, et al., 2006), suggesting that the temporal 
limits for shifting spatial attention may be different for symbolic and peripheral cues. However, in that study the instructions to shift attention diverged from the types of cues usually used in many attentional cueing paradigms, and it was not experimentally confirmed that participants shifted attention to the expected location following the endogenous cues (see Discussion for more detail). Thus, it is currently still unclear as to whether exogenous or endogenous attentional deployment differs in terms of their temporal dynamics with respect to spatial distance.

We here ask whether the time course of allocating spatial attention exogenously or endogenously is dependent upon spatial distance. We used a cueing paradigm that employs traditional spatial attention cues and that allows to measure the timing of attentional allocation directly, while controlling for general task difficulty (i.e., target processing time, response difficulty, etc.). We expected that endogenous attentional deployment would take longer than exogenous attention overall, as previous research indicates (Nakayama \& Mackeben, 1989; Chakravarthi \& van Rullen, 2011). The main question of interest was whether exogenous and endogenous attention show differences in their temporal dynamics as a function of spatial distance. If spatial distance affects their respective time courses differently, this would support a model in which exogenous and endogenous attentional deployment are supported by different processes that place distinct temporal constraints on each type of attention. Alternatively, if both show a similar relationship between the time course and spatial distance, this would suggest that the deployment of exogenous and endogenous attention shares similar temporal limits. Consistent with the latter account, we find that both endogenous and 
exogenous attention are slower when deployed to far spatial locations relative to nearby locations.

\section{Methods}

\section{Experiment 1: Measuring temporal limits of spatial attention shifts using orientation}

Data for all experiments is available at:

https://osf.io/tc8n4/?view_only=0a19156a906644029842fb8c316b1959

Participants: Across all experiments, participants were between 18 and 28 years of age and had normal or corrected-to-normal vision and normal color vision. All participants provided written informed consent in accordance with the Institutional Review Board at UC San Diego prior to participation. Eye movements were monitored with electrodes placed next to the left and right eyes, and trials with eye movements were excluded from the main analysis (for more details, see Horizontal Eye Movement Recordings). Across all experiments, we excluded full data sets from participants if they consisted of less than 40 trials in any of the conditions after artifact rejection.

Nineteen participants completed Experiment 1. Data from one participant were excluded from the main data analysis because more than 20 trials were rejected due to eye movements in one of the conditions, leaving less than the 40 -trials cut-off. The number of participants for Experiment 1 was chosen based on pilot data in the laboratory that suggested that we need 14 participants to detect a medium-sized effect $(\eta 2=.3)$ for spatial distance, which would be the 
smaller effect relative to the effect of cue type ( $\eta 2=.6$ based on pilot data). After data analyses was completed, we calculated the power we had to detect the main effects of interest with our final sample size: 1) The power for the effect of cue type (endogenous vs. exogenous) was 0.95;

2) The power for the effect of spatial distance was 0.76 . This indicates that we were overall sufficiently powered to detect the effects of interest.

Stimuli and Procedure: Participants were seated with a viewing distance of approximately $57 \mathrm{~cm}$ from a CRT monitor ( $85 \mathrm{~Hz}$ ). Throughout the experiment, the background of the display was gray (RGB: $127,127,127)$ with a dark gray fixation cross in the center (RGB: $50,50,50 ; 0.5^{\circ}$ by $0.5^{\circ}$ of visual angle). The display consisted of four gray outlined circles (line thickness: $0.2^{\circ}$ of visual angle; RGB: 100, 100, 100) presented at four locations: top left, top right, bottom right, bottom left (Figure 1a). Each circle contained an oriented black triangle (RGB: 0, 0, 0). Across trials, these stimuli were either presented nearby fixation (midpoint of stimuli was $5^{\circ}$ of visual angle from fixation; near condition) or far in the periphery (midpoint was $18^{\circ}$ of visual angle from fixation; far condition). The diameter of the nearby circles was $2.0^{\circ}$ (triangles: $1.7^{\circ}$ long and $0.34^{\circ}$ maximum width of visual angle), and $4.5^{\circ}$ for the distant circles (triangles: $4.2^{\circ}$ long and $0.84^{\circ}$ maximum width of visual angle) to account for less accurate vision in the periphery. On each trial, a random orientation $\left(0\right.$ to $\left.360^{\circ}\right)$ was picked for one of the triangles, and the other three triangle orientations were chosen to be $90^{\circ}$ apart from each other; the orientations were randomly assigned to the four locations. Thus, each triangle had a unique orientation at the beginning of each trial, and starting orientations varied from trial to trial. As soon as a trial began, each triangle started rotating continuously, changing its orientation by $2^{\circ}$ every frame 
(every $11.76 \mathrm{~ms}$ ). The direction of rotation (clockwise or counterclockwise) was randomly and independently determined for each oriented triangle on each trial.

After a random time period of $1,300-1,600 \mathrm{~ms}$ (uniform distribution), an attention cue was presented for $\sim 106 \mathrm{~ms}$ (9 frames). Participants were instructed to direct their attention to the location of the cue and remember the orientation of the triangle at the moment of the cue to report it at the end of the trial. The triangles continued to rotate for $1,100-1,400 \mathrm{~ms}$ after the cue, such that each trial was $2,800 \mathrm{~ms}$ long overall. Then, all triangles disappeared and the outline of the cued circle was highlighted $\left(0.4^{\circ}\right.$ line thickness) and a small gray dot (RGB: 100 , 100,$100 ; 0.6^{\circ}$ by $0.6^{\circ}$ ) appeared in the center of the previously cued circle for $500 \mathrm{~ms}$. Next, participants were required to report the orientation they saw at the moment of the cue by using a response wheel to adjust the orientation of a test triangle. The test triangle started off at a random angle, and once participants started moving the response wheel the test triangle changed orientation to match the orientation the participant chose with the response wheel. When participants identified the orientation that matched the orientation they saw at the time of the cue, they locked their response by clicking on the response wheel. All cues predicted the tested location with $100 \%$ validity, but the cues differed in their appearance to match traditionally used cues in endogenous and exogenous cueing paradigms. On one third of the trials the attention cues were central symbolic cues, and on another third of the trials the attention cues were peripheral salient cues (Figure 1b). The central symbolic cues (endogenous attention condition) consisted of four oriented gray lines $\left(1^{\circ} \times 0.1^{\circ} ; \mathrm{RGB}: 50,50\right.$, 50) presented next to the fixation cross ( $1^{\circ}$ distance), referencing the four stimulus positions (at $45^{\circ}, 135^{\circ}, 225^{\circ}, 315^{\circ}$ around fixation). During the cueing period, one of the lines turned white 
(RGB: $255,255,255)$, indicating to the participant that they should direct their attention to the location the line pointed to and remember the orientation at the time of the cue. The peripheral cue (exogenous attention condition) was a white ring (RGB: $255,255,255)$ that appeared around one of the oriented triangles to prompt participants to attend and remember the orientation at the same location.

The remaining third of trials served as a baseline condition to control for general memory- and response-related processes of the task. In particular, a single oriented rotating triangle that either matched the size of the "near location" condition (surrounding circle $2^{\circ}$ of visual angle) or "far-location" condition (surrounding circle $4.5^{\circ}$ of visual angle) was presented in the center of the screen. A white ring flashed around the oriented triangle at an unpredictable time $(1,300-1,600 \mathrm{~ms}$, see above), and participants were asked to report the orientation they saw at the moment of the bright circle. Thus, participants similarly monitored a rotating oriented triangle, needed to extract a particular orientation at a discrete point in time, protect that representation from interference of the continuing rotation, and finally indicate the orientation using continuous report. Thus, the only difference between this baseline condition and the attention conditions was that attention did not need to be shifted to a new location. Thus, by subtracting out performance in this baseline condition, we can estimate the temporal dynamics of exogenous and endogenous attention with no influence of other general task factors. The different attention conditions (endogenous, exogenous, baseline) were blocked, and the spatial distance conditions (near, far) and cue locations (top left, top right, bottom left, bottom right) varied on a trial-by-trial basis. All conditions were counterbalanced across the 
experiment. Participants completed 15 blocks overall with 24 trials each, for an overall 60 trials in each attention-distance condition.

Horizontal Eye Movement Recordings: The horizontal electro-oculogram was acquired using a bipolar pair of electrodes positioned at the external ocular canthi and data were digitized using an ActiCHamp amplifier (Brain Products $\mathrm{GmbH}$, Germany). Data were epoched into trials and artifact rejection was performed for the interval -100 to $+2800 \mathrm{~ms}$ (the total trial time until response). Each trial was visually inspected for eye movements, and trials were removed from the final analysis if the eyes deviated from fixation.

Analysis: We estimated the time course for the deployment of spatial attention by calculating the difference in degrees between a given response and the degrees shown at the onset of the attention cue. Then, we multiplied this difference by the time it took the triangle to rotate. For example, if during the time of the cue the triangle was oriented $35^{\circ}$, and a participant reported an orientation of $60^{\circ}$, this would result in a difference of $25^{\circ}$ (the response 'error'). As the triangle rotated $2^{\circ}$ every $\sim 11.76$, this would indicate a temporal difference of $\sim 146 \mathrm{~ms}$. We estimated this temporal delay for each condition separately using an individual's median response error for each condition (endogenous/exogenous; near/far, collapsed across the four locations), and then subtracted the corresponding baseline conditions for each individual. In particular, we subtracted the small-stimulus baseline from the close-location conditions, and the large-stimulus baseline from the far-location conditions. The main results reported in the paper are focused on the data post baseline subtraction, but the detailed results of the baseline 
conditions are reported in Table 1. Critically, the pattern of results does not change if we omit this baseline subtraction.

\section{Results Experiment 1}

The results of Experiment 1 show that attentional deployment took overall longer for endogenous cues $(370 \mathrm{~ms},+/-53)$ relative to exogenous cues $(110 \mathrm{~ms}+/-43)$, and that attention was slower when deployed at more distant locations relative to locations nearby fixation (endogenous: $406 \mathrm{~ms}$ vs. $332 \mathrm{~ms}$; exogenous: $141 \mathrm{~ms}$ vs. $80 \mathrm{~ms}$; see Figure $1 \mathrm{c}$ ). A repeatedmeasures analysis of variance (ANOVA; alpha level 0.05) with factors cue type (exogenous, endogenous) and spatial distance (near, far) confirmed this statistically, revealing a main effect of cue type $(F(1,17)=50.21 ; p<0.000001 ; \eta 2=.75)$, and a main effect of spatial distance $(F(1,17)$ $=12.2, p=.003 ; \eta 2=.42)$. There was no interaction $(p=.56 ; \eta 2=.02)$. A priori planned follow-up paired t-tests indicated that there was a reliable spatial distance effect for exogenous cues $(t(17)=3.02, p=.008 ; \eta 2=.35)$ and endogenous cues $(t(17)=3.11 ; p=.006 ; \eta 2=0.36)$ of similar effect size. Overall, this suggests that it takes longer to deploy spatial attention to locations further away from current fixation, independent of how attention was initiated. 
a)

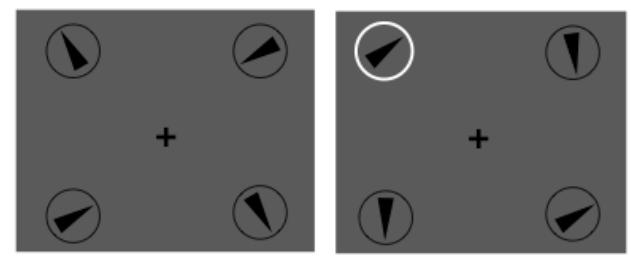

1300-1600ms

$100 \mathrm{~ms}$

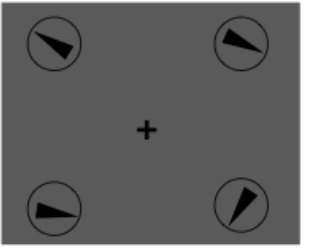

1100-1400ms

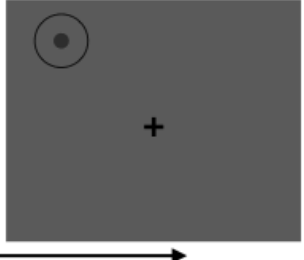

Probe \&

continuous report b)

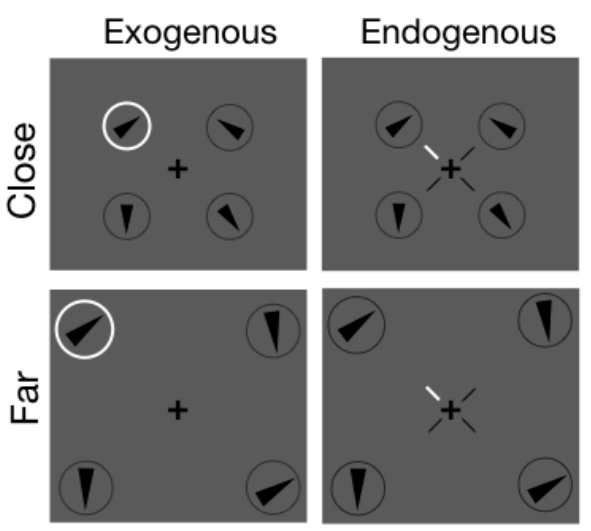

c)

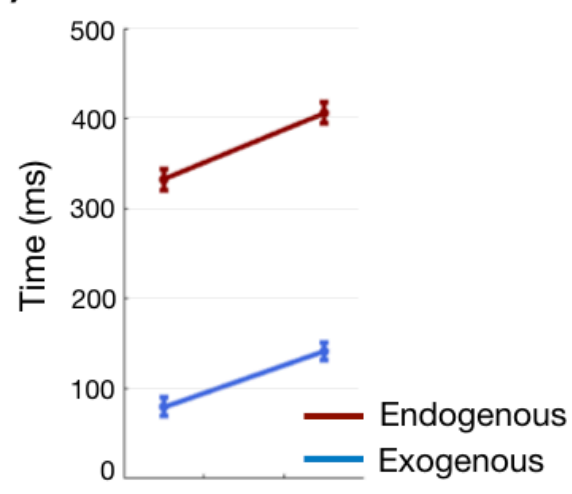

Figure 1. Trial structure, conditions, and results of Experiment 1. a) Each trial began with four unique and randomly chosen oriented triangles presented at four locations. Each of the triangles rotated through orientation space throughout the trial. At an unpredictable time, one location was cued (example shows exogenous cue) for a brief moment and participants were instructed to report the orientation they saw at the moment of the cue at the end of the trial using continuous report. b) The triangles were either presented at four locations close to the central fixation (top row) or far (bottom row), and cues were either peripheral flashes (left; exogenous) or central symbolic lines (right; endogenous). c) The estimated attentional shift 
time was slower for endogenous relative to exogenous cues; of most interest, attentional deployment was slower for more distant locations, regardless of cue.

\section{Experiment 2: Measuring temporal limits of spatial attention shifts using color space}

Experiment 1 suggests that the temporal dynamics of both endogenous and exogenous attentional allocation depend on the spatial distance attention has to travel: When attention is deployed to a location nearby fixation it is faster relative to when attention is deployed to more distant locations. In Experiment 1 we used continuously rotating triangles and asked participants to report the orientation at the moment of a cue. Given that participants have meta-knowledge about orientation space - i.e., know that 45 degrees must have happened before 60 degrees, participants could in principle consider the temporal delay of shifting attention to the cued location and adjust their response to try to account for the delay. To reduce the possibility of such a strategy, and to replicate the previous results using a modified design, we next used continuously changing colors that rotated through hue space (instead of orientation space). Presumably, naïve participants have less knowledge about the organization of color space than orientation space, making it thus less likely that they try to explicitly account for temporal delays at response.

\section{Methods}


Participants: Eighteen participants took part in Experiment 2. Data from two participants were excluded from the main data analysis because more than 20 trials were rejected due to eye movements in one of the conditions. After data analyses was completed, we calculated the power we had to detect the observed effects with our final sample size: 1) The power to detect the main effect of cue type (endogenous vs. exogenous) was 0.99; 2) The power to detect the main effect of spatial distance was 0.79 .

Stimuli and Procedure: The procedure was very similar to Experiment 1 with a few exceptions (see Figure 2a). The background of the display was black [RGB: $0,0,0$ ] with a gray fixation cross in the center (RGB: $100,100,100 ; 0.5^{\circ}$ by $0.5^{\circ}$ of visual angle). The display consisted of four disks, just like in Experiment 1, but each disk was uniquely colored at the beginning of each trial and then slowly and continuously changed color by rotating through $\mathrm{CIE} L^{*} a * b$ space (centered at $L=54, a=21.5, b=11.5)$. In particular, on each trial a random color value was chosen from a $360^{\circ}$ color wheel for one disk, and the remaining three colors were chosen to be $90^{\circ}$ apart from that color. These four starting color values were randomly assigned to the four disks. The colors changed continuously for each disk by rotating through the $\mathrm{CIE}$ lab color wheel by $1^{\circ}$ every frame. The direction of movement through color space (clockwise or counterclockwise) was randomly and independently determined for each disk on each trial.

The attention cues and conditions were identical to Experiment 1. Once a test probe appeared, participants were required to respond by using a response wheel to adjust the color of the circle to the color they saw during the time of the cue. The test circle continuously showed the color as the participant was choosing it. Critically, participants were never exposed to the color 
wheel itself. The response color started off at a random point in CIE lab color space and participants locked their response by clicking on the response wheel.

\section{Results Experiment 2}

The results showed that attentional shift times were dependent on the type of cue and the spatial distance: Endogenous attentional shift times were overall slower $(402 \mathrm{~ms},+/-52)$ relative to exogenous attentional shift times $(274 \mathrm{~ms}+/-73 \mathrm{~ms})$, and longer distances required more time than shorter distances (endogenous: $440 \mathrm{~ms}$ vs. $366 \mathrm{~ms}$; exogenous: $327 \mathrm{~ms}$ vs. $222 \mathrm{~ms}$; see Figure 2b). A repeated-measures ANOVA with factors cue type (exogenous, endogenous) and spatial distance (near, far) revealed a main effect of cue type $(F(1,15)=63.16 ; p<.000001$; $\eta 2=.81$ ), indicating that participants shifted attention faster on exogenous relative to endogenous cues. Furthermore, there was a main effect of spatial distance $(F(1,15)=9.17, p=$ $.01 ; \eta 2=.38)$, but no interaction ( $p=.35 ; \eta 2=.06)$, suggesting that allocating spatial attention to far spatial locations took longer relative to close spatial locations. Planned follow-up t-tests showed that there was a reliable distance effect for exogenous cues $(t(15)=2.69, p=.02$; $\eta 2=.32)$, as well as for endogenous cues $(t(15)=2.76 ; p=.02 ; \eta 2=.34)$, again with a similar effect sizes.

These results replicate the findings of Experiment 1, further suggesting that exogenous and endogenous attention show similar dependencies in terms of their time course for shifting attention to close and far locations. Importantly, they show that even when participants are relatively unfamiliar with the organization of the feature space used to probe attentional shift 
times, they show similar spatial distance effects, suggesting that the results are not due to response biases.

a)

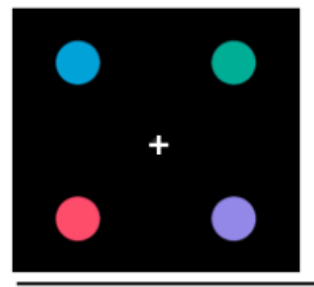

$1300-1600 \mathrm{~ms}$

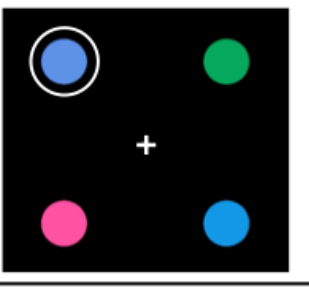

$100 \mathrm{~ms}$

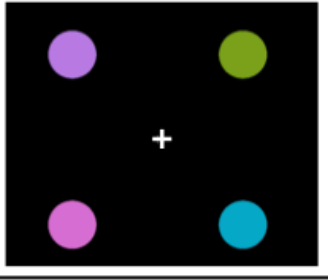

$1100-1400 \mathrm{~ms}$

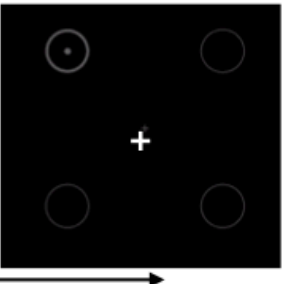

Probe \& continuous report b)

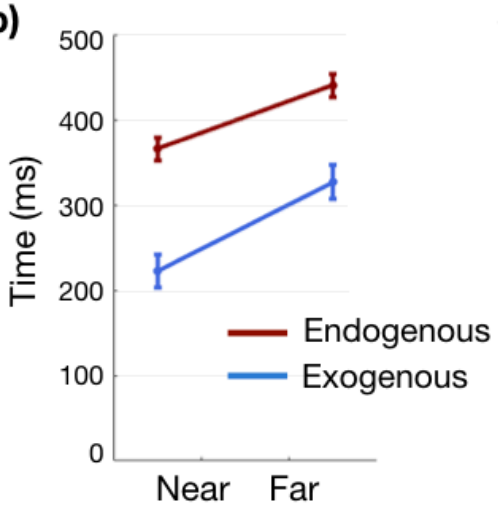

c)

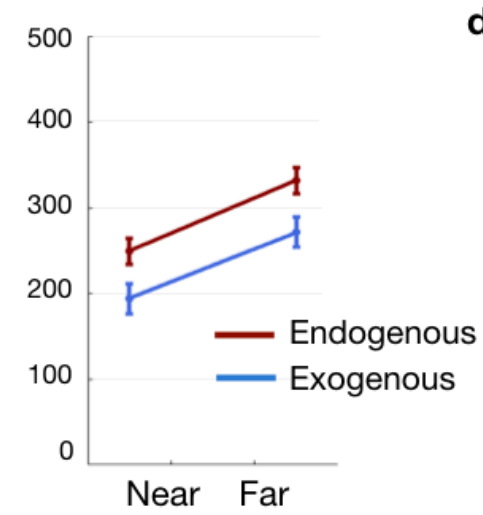

d)

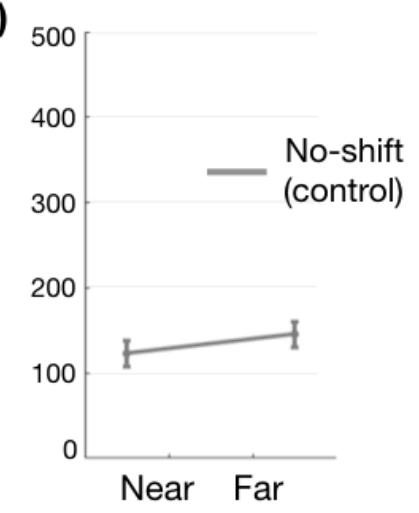

Figure 2. Example trial structure and results of experiments 2-4. a) In Experiment 2, each trial began with four unique and randomly chosen colors presented at four locations. The colors continuously changed throughout the trial by moving through CIE lab color space. At an unpredictable time, one location was cued (example shows exogenous cue) for a brief moment and participants were instructed to report the color they saw at the moment of the cue at the end of the trial using continuous report. b) Results of Experiment 2 show slower attentional shift times for endogenous relative to exogenous cues, and for locations further away relative 
to closer to central fixation. c) Results of Experiment 3, in which the near and far locations were along the horizontal meridian also show that attentional allocation is slower for endogenous cues and far distances. d) A control experiment which did not require attentional shifting found no effect of spatial distance on reporting the color near or far from fixation.

\section{Experiment 3: Measuring temporal limits of horizontal spatial attention shifts}

The first two experiments suggest that allocating spatial attention to locations further away from current fixation takes longer relative to allocating attention to nearby locations. However, in both cases the target locations appeared in each of the visual quadrants, requiring diagonal shifts of attention. In many classic attentional cueing paradigms, attentional shifts occur horizontally - from left to right or vice versa. To check whether there is anything particular about diagonal vs. horizontal attention shifts, we next simplified the task and only tested near and far locations along the horizontal meridian.

\section{Methods}

Participants: Twenty participants completed the experiment. Data from two observers were removed due to less than 40 trials in one of the conditions. A subsequent power analysis showed that we had the following power to detect the observed effects given our sample size: 1) The power to detect the effect of cue type (endogenous vs. exogenous) was $0.90 ; 2$ ) the power to detect the effect of spatial distance 0.77 . 
Stimuli and Procedure: The stimuli and procedures were identical to Experiment 2, except that only two disks were shown on each trial. During the near location condition, the two disks were shown at $5^{\circ}$ eccentricity to the left and right of fixation, and in the far location condition at $18^{\circ}$ eccentricity to the left and right of fixation. The cues were adjusted accordingly.

\section{Results Experiment 3}

We again found that attentional shifts times were overall slower for endogenous (290ms $+/-58)$ relative to exogenous attention $(233 \mathrm{~ms},+/-54)$, and that it took longer to shift attention to the more distant location in both conditions (endogenous: $332 \mathrm{~ms}$ vs. $250 \mathrm{~ms}$; exogenous $272 \mathrm{~ms}$ vs 194ms). Statistical analyses confirmed this observation revealing a main effect of cue type $(F(1,17)=22.21, p=.0002, \eta 2=.57$, and a main effect of spatial distance $(F(1,17)=6.85 ; p=.02$, $\eta 2=.29$, but no interaction $(p=.850 ; \eta 2=.002)$. Follow-up paired $t$-tests revealed that the distance effect was reliable for exogenous attention $(t(17)=2.2, p=.04 ; \eta 2=.22)$ and endogenous attention $(t(17)=2.67, p=.01, \eta 2=.30)$. Thus, when spatial attention was shifted along the horizontal meridian - similar to what is usually done in many attentional cueing studies, it was slower to allocate attention to a more distant relative to a nearby location, regardless of whether attention was triggered exogenously or endogenously. 


\section{Experiment 4: Control experiment for color report at near and far eccentricities}

Thus far, the results point to a consistent spatial distance effect on the time course for both exogenous and endogenous attentional deployment. Across three experiments, we observed that the feature reported was delayed relative to the cue appearance more so for locations far away than locations nearby. Are these differences really due to attentional deployment, or just a general effect of performing the orientation/color report task at closer and farther eccentricities? While we adjusted the size of the stimuli to adjust for perceptual differences across eccentricities (M-scaling; Cowey \& Rolls, 1974), it could still be the case that accurately representing an orientation or color closer to the fovea vs. more peripherally was not accounted for by our adjustment. To check this, we ran a control experiment with no attention cues. Specifically, participants only monitored a single disk (while maintaining fixation), and were asked to report the color of that disk at an unpredictable time. We varied spatial location (near, far) and also included a baseline condition, but removed all attention cues altogether.

\section{Methods}

Participants: Twenty-one participants took part in the experiment. One participant did not finish the task, and data from two subjects had to be removed because of an excessive amount of eye movements ( $<40$ trials with no eye movements in one of the conditions). We chose the number of participants based on Exp. 3, which most closely resembled the stimulus set up. Note that we only compared two conditions in Exp. 4 (close vs. far). Assuming the same effect size for that comparison in Exp. 3, we calculated that we would need 17 participants to achieve 
a power of 0.8 to detect such an effect. Note that we had double the number of trials in each condition in this experiment (see below), and thus likely underestimated our power.

Stimuli and Procedure: The stimuli and procedures were similar to Experiments 2 with the following exceptions. On each trial, participants only saw a single colored disk at either one of the near or far locations. At a random moment in time the disk disappeared and the participant had to report the last color they saw using continuous report. Thus, participants had to maintain spatial attention at the disk location the entire time and no attentional shifts were involved. Spatial distance was randomly varied trial by trial. On $1 / 3$ of trials the disks appeared in the center of the screen to serve as the baseline condition, just as in previous experiments. Participants completed 15 blocks of 24 trials each; note that since there were no different attention conditions, there were overall 120 trials for each eccentricity condition.

\section{Results}

A paired t-test with eccentricity (close, far) as a factor indicated no statistically reliable difference between the two eccentricity conditions (close: $123 \mathrm{~ms}$ vs. far: $145 \mathrm{~ms} ; t(17)=.74, p=$ $.47, \eta 2=.03$; Scaled JZS Bayes Factor $=3.22$; Rouder et al., 2009), suggesting that general task performance was the same across the different eccentricities.

\begin{tabular}{|c|c|c|c|c|}
\hline & Small & Large & p-value & $\eta 2$ \\
& stimulus & stimulus & & \\
\hline
\end{tabular}




\begin{tabular}{|l|c|c|c|c|}
\hline Exp 1- orientation & $200.00 \mathrm{~ms}+/-$ & $176.80 \mathrm{~ms}$ & .13 & 0.13 \\
& 27.24 & $+/-28.54$ & .22 & .10 \\
\hline Exp2 - color diagonal & $270.96 \mathrm{~ms}$ & $293.38 \mathrm{~ms}$ & .29 \\
\hline Exp 3- color horizontal & $196.73 \mathrm{~ms}$ & $239.22 \mathrm{~ms}$ & .02 & .29 \\
& $+/-22.27$ & $+/-22.57$ & & \\
\hline Exp 4- color control & $12.88 \mathrm{~ms}+/-$ & $26.73 \mathrm{~ms}$ & .28 & \\
& $8.81 \mathrm{~ms}$ & $(+/-$ & & \\
& & $16.02 \mathrm{~ms})$ & & \\
\hline
\end{tabular}

Table 1: Median response times in the baseline conditions for small and large stimuli for all experiments. These baseline times were subtracted from each of the estimated orienting times reported in the main analysis, separately for each stimulus size. Note that the statistically significant difference of Exp. 3 shows a pattern opposite of our main effect of spatial distance, thus if anything, makes us underestimate the effect of spatial distance in the main results. Also note that all main results hold if we do not subtract the baseline times.

\section{Discussion}

Across three experiments we measured how long it takes for exogenous and endogenous attention to be deployed at close and far locations from central fixation. We estimated 
attentional shift times by asking participants to report a specific feature value (orientation or color) of a stimulus that continuously moved through feature space at a constant speed. Specifically, participants were instructed to deploy attention following either a peripheral cue (exogenous) or a central cue (endogenous) and report the feature value they saw at the moment of the cue. We compared their responses to the feature value that was presented at the time of the cue and estimated the shift time based on the response error. To control for other task factors, such as remembering the feature until response or integrating continuously changing features over time, we added a control condition with no spatial attention shifts and subtracted performance on that task from the attention conditions. Using this continuous feature task, we found that endogenous attention was overall slower relative to exogenous attention, replicating previous research (Rabbit \& Müller, 1989; Keefe \& Störmer, 2020; Chakravarthi \& van Rullen, 2011). Critically, we found that attentional deployment times were dependent on spatial distance for both exogenous and endogenous attention. Specifically, it took longer for attention to be allocated at far locations relative to near locations, and this effect was of similar magnitude across both attention conditions. Importantly, we also showed that the difference in behavioral performance for close and far locations was not driven by lowlevel differences in perceiving the stimuli at close or far distances: when attention was already deployed at the location of the stimulus and no attentional shift was required, performance did not differ across the spatial distances (Exp. 4). Overall, these data suggest that the time to deploy attention depends on the spatial distance of target locations for both exogenously and endogenously driven attention, pointing to shared temporal limits across both attention types. 
Our results are broadly consistent with another recent study showing that endogenous attentional shift times depend on spatial distance, though that study found only minimal effects of spatial distance for exogenous attention (Chakravarthi \& van Rullen, 2011). There are several factors that can account for these differences, including the exact stimuli and subtraction methods used. In the previous study eight disks with rotating oriented lines ('clocks') were presented along a circle. To estimate endogenous attentional shift times, participants were first cued exogenously to one of the clocks (with a brief flash surrounding that clock), and then asked to move attention from that location 1,2 , or 5 steps away along the circle. To estimate exogenous attentional shift times, participants were instructed to first attend to an endogenously cued clock (the cue was a centrally presented line pointing to one location), and then shift attention to a subsequently exogenously cued location $(1,2$, or 5 steps away along the circle). The time it took for attention to shift to the first location (either by a peripheral exogenous cue or by a central endogenous cue) was subtracted from the estimated shift times in each condition, thereby effectively controlling for cue and target processing times. However, we believe there are two potential reasons why this design might have overestimated distance effects for endogenous attention while underestimating distance effects for exogenous attention. First, in the endogenous condition participants were instructed to shift attention a certain number of steps along the circle - which likely requires additional counting or planning processes that are different from processes involved when following more traditional endogenous cues such as a line or arrow (as used in our study), thus possibly overestimating shift times. Second, in the exogenous condition, it is unclear whether participants deployed attention effectively to the initially (endogenously) cued location. Instead, it seems plausible 
that they maintained attention in the center-at least on some of the trials - in which case the resulting spatial distances for exogenous cues would all be the same, leading to an underestimation of spatial distance effects on exogenous shift times. Thus, the discrepancies in magnitude of the spatial distance effects across studies - in particular for exogenous attention - are likely due to less sensitive measures in the previous study relative to ours. However, as the previous study found spatial distance effects overall, those results together with ours indicate that both types of attention are sensitive to the spatial distance attention moves. What processes underlie the distance-dependent time courses for exogenous and endogenous attention? One possibility is that these temporal dependencies arise from overlapping neural substrates that are involved in planning and initializing spatial attention shifts. Previous fMRI studies have found partially overlapping brain networks involved in the orienting of exogenous and endogenous attention (Peelen et al., 2004; Corbetta \& Shulman, 2002; Serences \& Yantis, 2007), consistent with the idea that top-down control signals that implement the spatial deployment are - at least in part -- shared for both types of attention. Within this framework, the distance-dependent temporal effects may be due to common processes that involve programming or planning the spatial shift, and would imply that these processes are distance-dependent, such that it takes longer to program the deployment of attention to far relative to nearby locations. This is also generally in line with eye movement data showing that saccade latencies are longer for more distant targets (Kalesnykas \& Hallett,1994; Zambarbieri, et al., 1995; but see Dafoe, et al., 2007 for no clear effects of eccentricity on saccade latencies), and that reflexive and voluntary saccades are programmed in parallel on a common motor map (Walker \& McSorley, 2006). Furthermore, other research 
indicates that spatial shifts of covert and overt attention - both when endogenously and exogenously triggered - share a network of frontoparietal and temporal brain regions that are responsible for programming and initializing spatial shifts (Grosbras, et al., 2005). Thus, the distance-dependent temporal effects we find here may arise during these earlier planning and programming stages that are shared across overt and covert, but also exogenous and endogenous attention.

Another possibility is that the common distance-dependent temporal effects are due to the architecture of the visual system, in particular the visual-spatial maps over which attention operates. Other research has shown that the limits of how many objects can be attended is constrained by the visual system's organization (Alvarez \& Cavanagh, 2005; Störmer, Alvarez, \& Cavanagh, 2014), and it may be the case that similar constraints are placed on the temporal dynamics of attentional orienting. In other words, it may take longer to deploy attention at locations that are represented at further distances from the fovea - possibly because of larger receptive fields at these locations and/or higher spatial uncertainty. As our task design does not allow to distinguish between spatial distance and eccentricity, it is plausible that the temporal signatures of exogenous and endogenous attention emerge because of the same underlying retinotopic map structure over which they operate. Previous work showing that endogenous attentional shift times are slower within a visual half-field relative to between visual half-fields is consistent with the notion that the visual system's architecture may limit the time course of spatial attention (Chakravarthi \& van Rullen, 2011). Future work could distinguish between these alternatives by independently varying eccentricity and spatial distance and/or use neural 
measures to directly assess the involvement of top-down control processes in higher-level regions and sensory enhancement in visual cortex.

The question of whether and how shifting spatial attention is dependent upon spatial distance has been of debate for several decades. The goal of many earlier studies was to test and compare 'analog' and 'quantile' models of attention, with the first assuming that spatial attention continuously moves across the visual field similar to a 'spotlight' (Egly \& Homa, 1991; Rizzolatti, et al., 1987; Shulman et al., 1979; Tsal, 1983), and the latter hypothesizing that attention is implemented in a discrete manner, essentially 'jumping' from one location to another (Sagi \& Julesz, 1985; Yantis, 1988; Cheal \& Lyon, 1989; Sperling \& Weichselgartner, 1995; Kwak, et al.,, 1991; Remington \& Pierce, 1984). Strict versions of a spotlight model assume that attention moves at a fixed velocity from one location to the other, and thus predict that it takes longer time to move further distances. While this account is broadly consistent with the data pattern observed here, it also predicts that processing of visual information is enhanced along the path attention moves - something we did not measure here because the main goal of our study was to compare the time courses of exogenous and endogenous attention as a function of spatial distance. Thus, while our data are consistent the spotlight metaphor in general, we do not think they speak to it directly.

Overall, our data reveal that exogenous and endogenous attention show similar temporal sensitivities with regards to where attention is deployed in the visual field. This may be because of the same underlying visual-spatial maps over which attention operates, or because of shared mechanisms that are involved in planning these shifts of attention, or both. Broadly our data show that once the initial time it takes to interpret the endogenous cue and 
map it to the physical location is accounted for, the temporal dynamics are strikingly similar across exogenous and endogenous attention. This suggests that the time courses of exogenous and endogenous attention are not independent per se, but instead suggest a shared temporal limit in terms of the spatial distance of to-be-attended locations.

\section{Acknowledgments}

We thank Audrey Barsczc and Celene Gonzalez for help with data collection, and Angus Chapman, Jonathan Keefe, and Jamal Williams for helpful comments on the project. 


\section{References}

Alvarez, G. A., \& Cavanagh, P. (2005). Independent resources for attentional tracking in the left and right visual hemifields. Psychological science, 16(8), 637-643.

Barbot, A., Landy, M. S., \& Carrasco, M. (2012). Differential effects of exogenous and endogenous attention on second-order texture contrast sensitivity. Journal of Vision, 12(8), 6-6.

Carlson, T. A., Hogendoorn, H., \& Verstraten, F. A. (2006). The speed of visual attention: What time is it?. Journal of Vision, 6(12), 6-6.

Carrasco, M. (2011). Visual attention: The past 25 years. Vision research, 51(13), 1484-1525.

Chakravarthi, R., \& VanRullen, R. (2011). Bullet trains and steam engines: Exogenous attention zips but endogenous attention chugs along. Journal of Vision, 11(4), 12-12.

Cheal, M., \& Lyon, D. (1989). Attention effects on form discrimination at different eccentricities. The Quarterly Journal of Experimental Psychology Section A, 41(4), 719-746.

Chica, A. B., Bartolomeo, P., \& Lupiáñez, J. (2013). Two cognitive and neural systems for endogenous and exogenous spatial attention. Behavioural brain research, 237, 107-123. 
Corbetta, M., \& Shulman, G. L. (2002). Control of goal-directed and stimulus-driven attention in the brain. Nature reviews neuroscience, 3(3), 201-215.

Cowey, A., \& Rolls, E. T. (1974). Human cortical magnification factor and its relation to visual acuity. Experimental Brain Research, 21(5), 447-454.

Dafoe, J. M., Armstrong, I. T., \& Munoz, D. P. (2007). The influence of stimulus direction and eccentricity on pro-and anti-saccades in humans. Experimental Brain Research, 179(4), 563-570.

Egeth, H. E., \& Yantis, S. (1997). Visual attention: Control, representation, and time course. Annual review of psychology, 48(1), 269-297.

Egly, R., \& Homa, D. (1991). Reallocation of visual attention. Journal of Experimental Psychology: Human Perception and Performance, 17(1), 142.

Eriksen, C. W., \& Hoffman, J. E. (1972). Temporal and spatial characteristics of selective encoding from visual displays. Perception \& psychophysics, 12(2), 201-204.

Fuller, S., Park, Y., \& Carrasco, M. (2009). Cue contrast modulates the effects of exogenous attention on appearance. Vision Research, 49(14), 1825-1837. 
Grosbras, M. H., Laird, A. R., \& Paus, T. (2005). Cortical regions involved in eye movements, shifts of attention, and gaze perception. Human brain mapping, 25(1), 140-154.

Hahn, B., Ross, T. J., \& Stein, E. A. (2006). Neuroanatomical dissociation between bottom-up and top-down processes of visuospatial selective attention. Neuroimage, 32(2), 842-853.

Hazlett, C. J., \& Woldorff, M. G. (2004). Mechanisms of moving the mind's eye: planning and execution of spatial shifts of attention. Journal of Cognitive Neuroscience, 16(5), 742-750.

Hopfinger, J. B., \& West, V. M. (2006). Interactions between endogenous and exogenous attention on cortical visual processing. Neurolmage, 31(2), 774-789.

Jonides, J. (1981). Voluntary versus automatic control over the mind's eye's movement. Attention and performance, 187-203.

Kalesnykas, R. P., \& Hallett, P. E. (1994). Retinal eccentricity and the latency of eye saccades. Vision research, 34(4), 517-531.

Keefe, J. M., \& Störmer, V. S. (2020). Lateralized alpha activity and slow potentials shifts over visual cortex track the time course of both endogenous and exogenous orienting of attention. bioRxiv, 2019-12. 
Kwak, H. W., Dagenbach, D., \& Egeth, H. (1991). Further evidence for a time-independent shift of the focus of attention. Perception \& psychophysics, 49(5), 473-480.

LaBerge, 1995; Attentional Processing: The Brain's Art of Mindfulness, Perspectives in Cognitive Neuroscience Series, Volume2 Harvard university Press

Ling, S., \& Carrasco, M. (2006). Sustained and transient covert attention enhance the signal via different contrast response functions. Vision research, 46(8-9), 1210-1220.

Müller, H. J., \& Rabbitt, P. M. (1989). Spatial cueing and the relation between the accuracy of "where" and "what" decisions in visual search. The Quarterly Journal of Experimental Psychology, 41(4), 747-773.

Nakayama, K., \& Mackeben, M. (1989). Sustained and transient components of focal visual attention. Vision Research, 29, 1631-1647. https://doi.org/10.1016/0042-6989(89)90144-2

Peelen, M. V., Heslenfeld, D. J., \& Theeuwes, J. (2004). Endogenous and exogenous attention shifts are mediated by the same large-scale neural network. Neuroimage, 22(2), 822-830.

Posner, M. I. (1980). Orienting of attention. Quarterly journal of experimental psychology, 32(1), 3-25. 
Remington, R., \& Pierce, L. (1984). Moving attention: Evidence for time-invariant shifts of visual selective attention. Perception \& Psychophysics, 35(4), 393-399.

Rizzolatti, G., Riggio, L., Dascola, I., \& Umiltá, C. (1987). Reorienting attention across the horizontal and vertical meridians: evidence in favor of a premotor theory of attention. Neuropsychologia, 25(1), 31-40.

Rouder, J. N., Speckman, P. L., Sun, D., Morey, R. D., \& Iverson, G. (2009). Bayesian t tests for accepting and rejecting the null hypothesis. Psychonomic bulletin \& review, 16(2), 225-237.

Serences, J. T., \& Yantis, S. (2007). Spatially selective representations of voluntary and stimulusdriven attentional priority in human occipital, parietal, and frontal cortex. Cerebral cortex, 17(2), 284-293.

Shulman, G. L., Remington, R. W., \& Mclean, J. P. (1979). Moving attention through visual space. Journal of Experimental Psychology: Human Perception and Performance, 5(3), 522.

Sperling, G., \& Weichselgartner, E. (1995). Episodic theory of the dynamics of spatial attention. Psychological review, 102(3), 503. 
Störmer, V. S., Alvarez, G. A., \& Cavanagh, P. (2014). Within-hemifield competition in early visual areas limits the ability to track multiple objects with attention. Journal of Neuroscience, 34(35), 11526-11533.

Störmer, V. S., \& Alvarez, G. A. (2016). Attention alters perceived attractiveness. Psychological Science, 27(4), 563-571.

Tsal, Y. (1983). Movement of attention across the visual field. Journal of experimental Psychology: Human Perception and performance, 9(4), 523.

Tsal, Y., \& L. Mevorach. (1991) "Effects of attention on length and distance estimates." Bulleting of the Psychonomic Society. Vol. 29. No. 6.

Walker, R., \& McSorley, E. (2006). The parallel programming of voluntary and reflexive saccades. Vision research, 46(13), 2082-2093.

Wright \& Ward, 2008 Orienting of Attention; Oxford New York Yantis, S. (1988). On analog movements of visual attention. Perception \& Psychophysics, 43(2), 203-206. 
Zambarbieri, D., Beltrami, G., \& Versino, M. (1995). Saccade latency toward auditory targets depends on the relative position of the sound source with respect to the eyes. Vision research, 35(23-24), 3305-3312. 